\title{
UNA ESPECIE NUEVA DE MYLABRIS FABRICIUS, 1775 (COLEOPTERA, MELOIDAE) DEL SURESTE DE LA PENÍNSULA IBÉRICA
}

\author{
J. L. Ruiz* y M. García-París**
}

\begin{abstract}
RESUMEN
Se describe una especie nueva de Mylabris Fabricius, 1775 de las montañas de la región sudoriental de la Península Ibérica (Macizo de la Sagra y áreas adyacentes, provincia de Granada). Mylabris deferreri sp. nov. se integra en el subgénero Micrabris por presentar un pronoto campaniforme, regularmente convexo, sin depresión transversa anterior; los tegumentos corporales negros; la placa mesosternal con "escudo" central bien patente, liso, brillante y glabro; y el edeago con los dos ganchos ventrales del lóbulo medio subiguales y manifiestamente alejados del ápice. En el ámbito ibérico, $M$. deferreri se aproxima fenéticamente a $M$. varians, $M$. dejeani y $M$. platai por presentar el punteado de la base de los élitros muy fino y apretado, en ocasiones prácticamente borrado, y la pubescencia de la misma zona muy corta, densa y erecta. La especie ibérica más afín a $M$. deferreri es $M$. varians, con la que comparte además una estructura similar de la protibia del macho y de la que se diferencia por la estructura de la genitalia masculina y el patrón de coloración elitral. Mylabris deferreri es la tercera especie de Mylabrini endémica de zonas relativamente elevadas de las Sierras Béticas del sur de España.
\end{abstract}

Palabras clave: Taxonomía, Especie Nueva, Península Ibérica, Mylabris, Micrabris, Meloidae, Coleoptera.

\section{ABSTRACT \\ A new species of Mylabris Fabricius, 1775 (Coleoptera, Meloidae) from the southeastern region of the Iberian Peninsula}

We describe a new species of Mylabris Fabricius, 1775, from the mountains of the southeastern region of the Iberian Peninsula (Macizo de la Sagra and surrounding areas, Province of Granada, Spain). Mylabris deferreri sp. nov. is included within the subgenus Micrabris based on the presence of a bell-shaped pronotum, smoothly convex, without anterior transverse depression; black tegument; mesosternal plate with a marked smooth and shiny central "shield"; aedeagus with the median lobe showing two subequal ventral hooks, clearly separated from the apex. Within the Iberian fauna of Micrabris, Mylabris deferreri is phenetically similar to $M$. varians, $M$. dejeani, and $M$. platai. These four species are characterized by a particular elytral basis, displaying very dense and fine shallow punctures, little marked, covered by very short and dense straight pubescence. Mylabris varians is likely related to $M$. deferreri, both sharing a similar structure of the male anterior tibiae. These two taxa differ by the shape of the male genitalia and the elytral coloration pattern. Mylabris deferreri is one of the three species of the tribe Mylabrini endemic to relatively high elevation areas of the Betic Mountains of southern Spain.

Key words: Taxonomy, New species, Iberian Peninsula, Mylabris, Micrabris, Meloidae, Coleoptera.

\footnotetext{
* Parques de Ceuta, Fase I, 3, 8-D, 51002 Ceuta. España. euserica@hotmail.com
}

** Museo Nacional de Ciencias Naturales. CSIC. José Gutiérrez Abascal, 2. 28006 Madrid. España. mcnp505@mcnc.csic.es 


\section{Introducción}

El género Mylabris Fabricius, 1775, incluye cerca de 180 especies distribuidas por la región Paleártica y el extremo noroccidental de la región Oriental (Bologna, 1991a; Bologna \& Pinto, 2002). La taxonomía del género es complicada y existen discrepancias en su tratamiento según autores, aunque actualmente se aceptan las propuestas de Pardo Alcaide (1948, 1950, 1954b, 1969) (aunque ver Kaszab, 1969) y especialmente la de Bologna (1991a) que reconoce la validez de 12 subgéneros. Uno de los subgéneros de Mylabris mejor caracterizados es Micrabris (especie-tipo, por designación original: Mylabris geminata Fabricius, 1798), creado por Kuzin (1954) y que incluye unas 40 especies localizadas principalmente en áreas de montaña, desde el Atlas de Marruecos hasta el norte de Birmania, con algunas especies esteparias, todas ellas limitadas al área meridional de la región paleártica (Bologna 1986, 1991a; Bologna et al., 1988).

Micrabris presenta, como el resto de representantes de la tribu Mylabrini, una morfología externa relativamente homogénea y una variabilidad intraespecífica considerable, sobre todo en el diseño cromático elitral (e.g. Pardo Alcaide, 1948, 1950; Bologna, 1991a; Bologna y Coco, 1991). Estos rasgos han propiciado la existencia de numerosas confusiones entre especies, en buena parte aclaradas por Pardo Alcaide (1948, 1954a, 1969) y Bologna (1979, 1986, 1991a, 1994) en el contexto europeo y circunmediterráneo.

En la Península Ibérica se conocen con certeza ocho especies de Micrabris, que a excepción de una, Mylabris (Micrabris) flexuosa Olivier, 1811, constituyen endemismos ibéricos con áreas de distribución más o menos restringidas (e.g. Pardo Alcaide, 1948, 1950, 1975; Bologna, 1991a). Mylabris (M.) geminata Fabricius, 1798, especie recientemente redefinida desde el punto de vista taxonómico y de distribución póntico-anatólicocentroasiática (Bologna, 1986, 1991a), ha sido repetidamente citada de provincias o regiones de la mitad norte peninsular o genéricamente de "España” (e.g. Heyden, 1870; Górriz, 1881, 1882; Champion, 1903; López-Neyra, 1914; Soumakov, 1915; Fuente, 1933). Según argumentan Pardo Alcaide (1948, 1950) y Bologna (1986, 1991a), estos registros se deben a confusiones con M. (M.) pauper (Escherich, 1899), y, por tanto, $M$. geminata ha de eliminarse del elenco de Mylabris ibéricos. La especie ibérica de más reciente descripción es Mylabris (Micrabris) platai Pardo
Alcaide, 1975, exclusiva de Sierra Nevada y formaciones béticas próximas (Sierra de Filabres y Sierra de Gádor) (Pardo Alcaide, 1975; Ruiz y Ávila, 1993), la cual según su autor (Pardo Alcaide, 1975) es posible que hubiera sido confundida anteriormente con Mylabris (Micrabris) varians (Gyllenhal, 1817).

En este trabajo, se describe una nueva especie de Mylabris del subgénero Micrabris, del macizo de La Sagra (provincia de Granada, Andalucía, sudeste de España), fenéticamente similar a $M$. varians, de la que se separa fundamentalmente por caracteres relativos a la genitalia masculina y al diseño elitral. Al igual que apuntó Pardo Alcaide (1975) para M. platai, cabe la posibilidad de que ésta especie haya pasado desapercibida hasta el momento por confusión con el taxon de Gyllenhal.

\section{Material y Métodos}

Se han estudiado 38 ejemplares de la especie que se describe, depositados en las colecciones del Museo Nacional de Ciencias Naturales de Madrid (MNCN), colección J.L. Ruiz (JLR) en Ceuta y colección J. de Ferrer (JF) en Algeciras (Cádiz), de los cuales 28 constituyen la serie típica. Asimismo, se ha estudiado abundante material de comparación de todas las especies ibéricas y norteafricanas de Micrabris, conservado en las colecciones citadas, y cuya enumeración sobrepasa los objetivos de esta contribución (Ruiz \& GarcíaParís, en prep.).

El estudio morfológico se efectuó sobre ejemplares conservados en seco, mediante observación con estereomicroscopio. Las genitalias, extraídas a ejemplares secos previamente rehidratados, se montaron en cartulinas con resina D.M.H.F., que se pincharon con su respectivo ejemplar. Las medidas se tomaron con micrómetro acoplado a uno de los oculares y los dibujos con cámara clara. La terminología de las diversas partes de la genitalia masculina se basa en la expresada por Pardo Alcaide (1948, 1950, 1954a, 1954b) y Bologna (1991a).

\section{Resultados}

\section{Mylabris (Micrabris) deferreri n. sp.}

HOLOTYPUS: 1 macho, etiquetado: "La Sagra (Granada), 2VII-1976, J. de Ferrer leg." (etiqueta blanca manuscrita) / "Holotipo, Mylabris deferreri Ruiz \& García-París, des. 2004" (etiqueta roja impresa); colección de Entomología del MNCN (n ${ }^{\circ}$ Catálogo Tipos: 9569). 

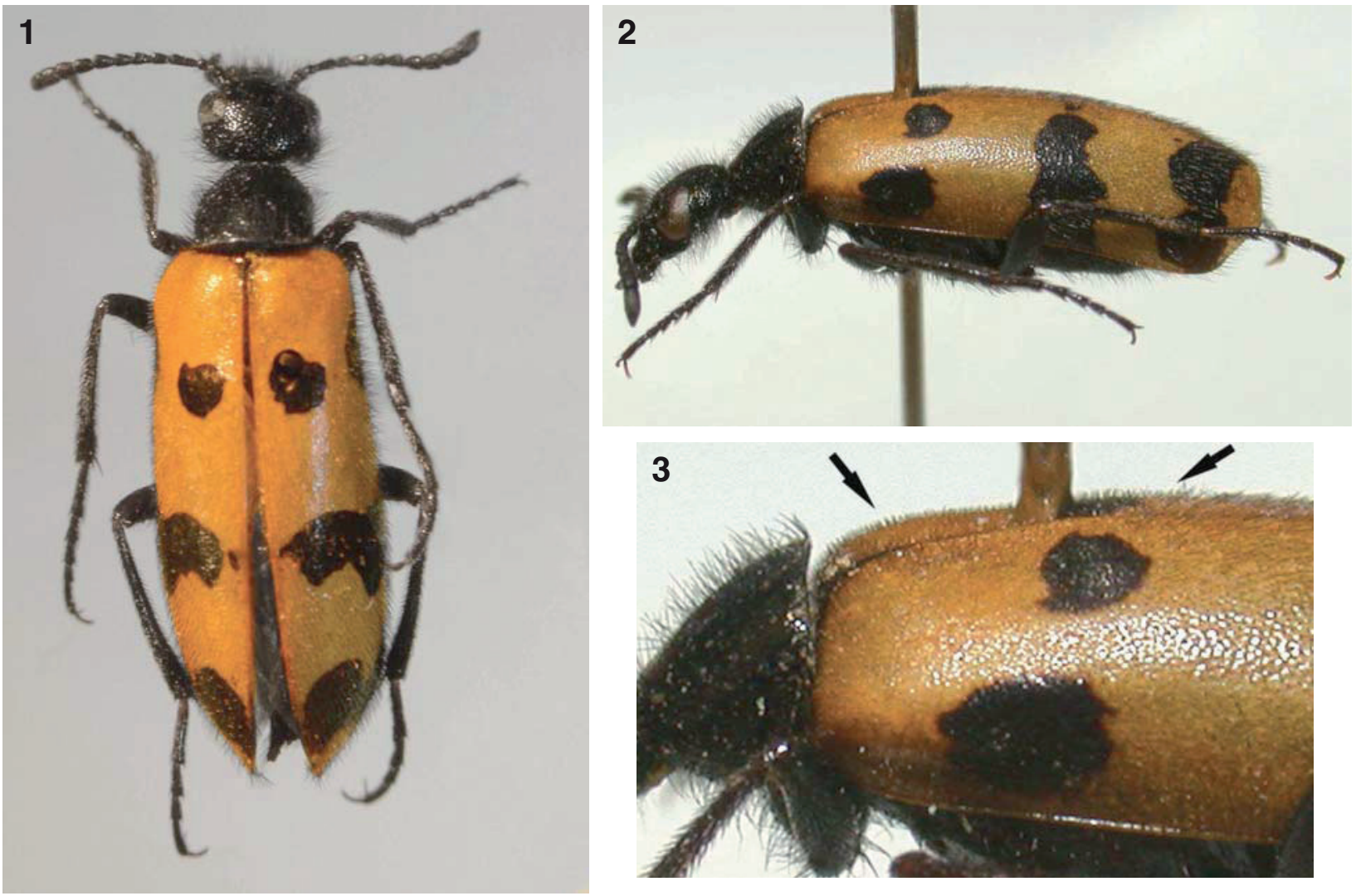

Figs. 1-3.- Mylabris (Micrabris) deferreri sp. nov. 1) Habitus en vista dorsal del holotipo. 2) Habitus en vista lateral del holotipo. 3) Vista lateral parcial del holotipo; las flechas señalan los dos tipos de pilosidad de los élitros: corta y erecta en el cuarto basal, más larga y tendida en el resto.

Figs. 1-3.- Mylabris (Micrabris) deferreri sp. nov. 1) Dorsal view of the holotype. 2) Lateral view of the holotype. 3) Partial lateral view of the holotype; arrows indicate different type of pubescence: short and straight on the basal fourth, lorger and postrated on the remaining surface.

PARATYPI: 27 ejemplares: 8 machos y 8 hembras, etiquetados: "La Sagra (Granada), Escalera 1900" (etiqueta blanca impresa); colección MNCN. 2 machos y 1 hembra, etiquetados: "La Sagra (Granada), 2-VII-1976, J. de Ferrer leg." (etiqueta blanca manuscrita); colección JLR. 2 machos y 2 hembras, etiquetados: "La Sagra (Granada), 3-VII-1976, J. de Ferrer leg." (etiqueta blanca manuscrita); colección JF. 1 hembra, etiquetada: "La Sagra (Granada), 2-VII-1976, J. de Ferrer leg." (etiqueta blanca manuscrita); colección MNCN. 2 hembras, etiquetadas: "La Sagra (Granada), 3-VII-1976, J. de Ferrer leg." (etiqueta blanca manuscrita); colección MNCN. 1 hembra, etiquetada: "Cabañuela - La Sagra, UTM 30S WH4005, 12-VII-1997, 1650 m, Pérez-López leg." (etiqueta blanca impresa); colección JLR. Todos ellos etiquetados: "Paratipo, Mylabris deferreri Ruiz \& García-París, des. 2004" (etiqueta roja impresa).

MATERIAL ADICIONAL EXAMINADO: Además de los ejemplares que constituyen la serie típica, se han examinado
10 ejemplares adscribibles a esta especie procedentes de: Galera (Granada), Escalera 1900 (7 ejemplares, Col. MNCN); Puebla de Don Fadrique (Granada), Escalera 1900 (2 ejemplares, Col. MNCN); La Sagra (Granada), Escalera 1900 (1 ejemplar sin cabeza, Col. MNCN).

Diagnosis: Se trata de una especie de Mylabris de pequeño tamaño y tegumentos corporales negros, incluida en el subgénero Micrabris por presentar un pronoto campaniforme, regularmente convexo, sin depresión transversa anterior; la placa mesosternal con "escudo" central bien patente, liso, brillante y glabro; y el edeago con los dos ganchos ventrales del lóbulo medio subiguales y manifiestamente alejados del ápice. Se agrupa con M. (Micrabris) varians y M. (M.) platai por carecer de pliegue longitudinal en el lado 
externo de la región inferior de la protibia del macho y por presentar el punteado de la base de los élitros muy fino y apretado, en ocasiones prácticamente borrado, y la pubescencia de la misma zona muy corta, densa y erecta. Estos caracteres de pubescencia también se presentan en $M$. (Micrabris) dejeani, especie que a diferencia de las anteriores presenta pliegue tibial. De todas estas especies se separa unequívocamente por la estructura de la genitalia masculina y el patrón de coloración elitral.

DESCRIPCIÓN DEL HOLOTIPO (MACHO): Longitud total: $9,8 \mathrm{~mm}$. Anchura máxima, situada a nivel del tercer cuarto posterior elitral: $2,8 \mathrm{~mm}$. Aspecto general estrecho, subparalelo y alargado (Fig. 1). Coloración general del cuerpo negra, muy brillante, tornándose castaño oscura en los tarsos y piezas bucales. Élitros amarillo anaranjados, ornamentados con una serie anterior constituida por dos puntos negros, y con dos bandas negras de contorno irregular, una media y otra posterior (Fig. 2). Pilosidad negra, larga y erizada en cabe$\mathrm{za}$, pronoto y región ventral, corta y tendida en los élitros, a excepción del cuarto basal donde es cortísima y erecta (Fig. 3).

Cabeza con tegumento negro y brillante, truncada por detrás; sienes subparalelas, ampliamente redondeadas en sus ángulos posteriores y ligeramente más largas que el diámetro transversal de los ojos, éstos son convexos pero poco salientes; sutura clípeo-frontal bien marcada y ligeramente arqueada; epistoma subtrapezoidal, transverso, doble de ancho que largo; labro transverso, subcordiforme, débilmente escotado en su borde anterior, 1,8 veces más ancho que largo, deprimido en el disco; frente ligeramente convexa, con una depresión central a nivel del cuarto superior de los ojos, de contorno subredondeado, en la cual se aprecian dos pequeñas manchas rojizas, difusas, convergentes hacia delante, así como dos pequeñas depresiones suboblicuas aledañas a la zona superior de las inserciones antenales; mandíbulas normales, con sus márgenes externos rectos en los dos tercios anteriores y curvadas en el tercio distal; maxilas y palpos normales, no modificados. Punteado relativamente fuerte, constituido por puntos gruesos e impresos, más denso a nivel de los dos tercios posteriores de la cabeza, vértex incluido, menos denso en la región anterior a la depresión centro-frontal que es lisa, con el punteado casi subrugoso a ambos lados e inmediatamente por encima de la depresión; epistoma con puntos algo menores que los de la frente y menos densos; labro con punteado muy escaso, similar al del epistoma. Pilosidad negra y erecta, más bien larga, inclinada hacia delante, y sigue el patrón de distribución de los puntos en los que se inserta.

Antenas de once artejos, largas, cuando están extendidas hacia atrás el último artejo alcanza la base del pronoto, moderada y paulatinamente dilatadas en maza alargada hacia la extremidad (Fig. 4); antenómero I dilatado en su zona distal y bruscamente estrechado en su tercio basal, doble de largo que el segundo; II corto y subgloboso; III estrecho y alargado, subparalelo; IV al VI subiguales en longitud y forma, ensanchados en el extremo, aproximadamente 0,63 veces la longitud del tercero; VII similar a los tres anteriores pero algo más largo y ensanchado en el extremo; VIII a $\mathrm{X}$ troncocónicos, cada vez más transversos y menos estrechados hacia la base; XI 2,1 veces más largo que el anterior, cilindrocónico, terminado en punta roma. Antenómeros I y II con pilosidad larga y semierecta; del II al VIII con pelos muy cortos e inclinados y del IX al XI cortísimos y tendidos; en los cuatro últimos el tegumento se torna mate y microrreticulado.

Pronoto dorsalmente convexo, sin depresión transversa anterior, igual de ancho que largo, con los márgenes laterales estrechados y redondeados hacia delante aproximadamente desde su mitad, muy débilmente estrechados hacia la base, ésta ligeramente arqueada a nivel del escutelo y con un reborde delgado. Punteado constituido por puntos gruesos similares o algo mayores que los de la cabeza, bien impresos y subcontiguos, con una estrecha línea media discal y dos pequeñas áreas laterales desprovistas de puntos. Pilosidad erizada, larga, similar a la de la cabeza, en su mayor parte inclinada hacia delante, siguiendo en general el mismo patrón de distribución de los puntos en los que se inserta.

Escutelo negro, pequeño, hemielíptico, anchamente redondeado por detrás y sin puntos.

Mesosterno (Fig. 5) convexo en la región media, con el escudo central grande, liso, lampiño y brillante. Mesopleuras con el borde anterior finamente aquillado y con la foseta o escotadura central bien marcada, sin ningún tipo de reborde interno.

Élitros amarillo anaranjados, con un diseño cromático constituido de la siguiente forma: (Figs. 1, 2): una estrecha lúnula basal negra, prácticamente cubierta por la base del pronoto, que se prolonga hasta una pequeña porción de la sutura elitral rodeando al escutelo; una serie anterior constituida por dos grandes manchas negras aisladas entre sí, la interna redondeada y la externa alargada pero sin 
4

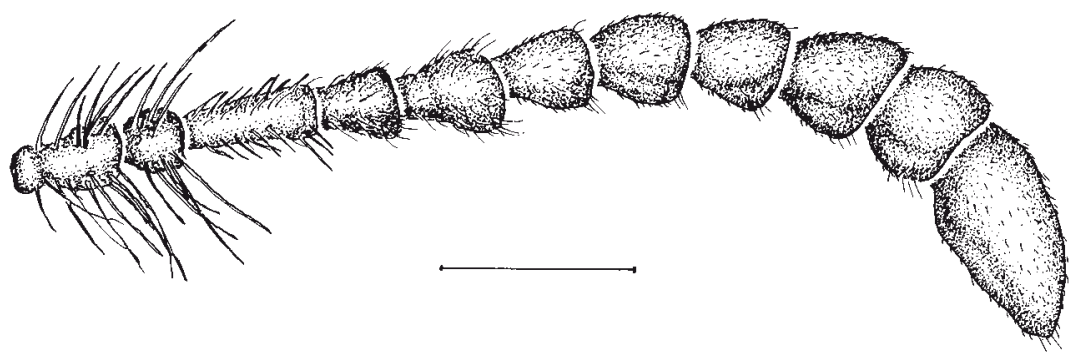

5

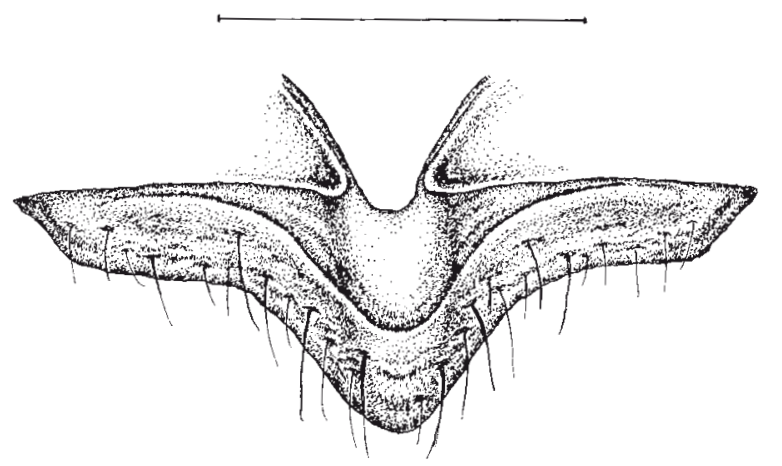

Figs. 4-5.- Mylabris (Micrabris) deferreri sp. nov. 4) Vista frontal de la antena del holotipo (J.L. Ruiz del.). 5) Vista ventral de la placa mesosternal (J.L. Ruiz del.). Barra de escala $=0,5 \mathrm{~mm}$.

Figs. 4-5.- Mylabris (Micrabris) deferreri sp. nov. 4) Frontal view of the antenna of the holotype (J.L. Ruiz del.). 5) Ventral view of the mesosternum plate of the holotype (J.L. Ruiz del.). Scale bar $=0,5 \mathrm{~mm}$.

llegar a interesar la región humeral; una ancha banda postmediana, irregular, prolongada hacia atrás en su mitad externa, que no llega a tocar ni la sutura ni el margen elitral externo; y por último, una banda subapical ancha, de contorno sinuoso, que alcanza tanto la sutura como el borde externo elitral. Carece de lúnula apical, a lo sumo sólo con el borde posterior estrechamente oscurecido. Base elitral muy finamente punteada, casi chagrinada, hasta la primera serie de puntos a partir de donde el punteado se va tornando paulatinamente más grueso y marcado, que alcanza su mayor tamaño en la región distal del élitro, pero siempre fino y subcontiguo. Pilosidad de los élitros cortísima y perpendicular en la región basal, a excepción de los márgenes laterales donde es más larga y erecta, haciéndose más larga y echada hacia atrás a partir de la primera serie de manchas negras.

Patas gráciles y alargadas, con pilosidad corta, apretada e inclinada. Tibias anteriores normales, sin pliegue longitudinal en su cara inferior, con una banda longitudinal de pelos amarillo-blanquecinos muy cortos y densos. Protarsómeros con un cepillito de cortos e hirsutos pelos blanquecinos en su región ventral. Espinas metatibiales cortas, estrechas e iguales.

Región ventral del cuerpo negra, brillante, con pubescencia alargada. Último esternito abdominal escotado en el medio de su margen posterior.

Edeago (Fig. 6a, b, c) con los parámeros estrechos y muy alargados, con sus lóbulos distales escasamente curvados en visión lateral, muy estrechos y de aspecto digitiforme en visión dorsal; falobase más bien estrecha en visión dorsal; lóbulo medio con los dientes subiguales (tipo isoharpagae, sensu Pardo Alcaide, 1948) muy alejados del extremo, y cercanos entre sí. Uncus del endofalo robusto.

VARIABILIDAD: Las hembras son similares a los machos, pero difieren de éstos por presentar las antenas más cortas y algo más mazudas, que 


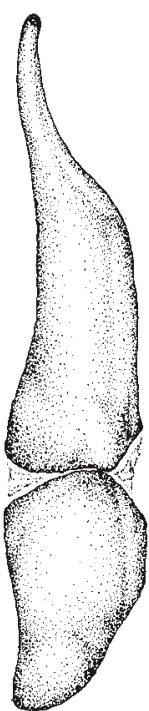

a

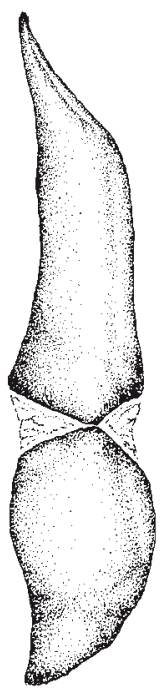

$\mathrm{g}$

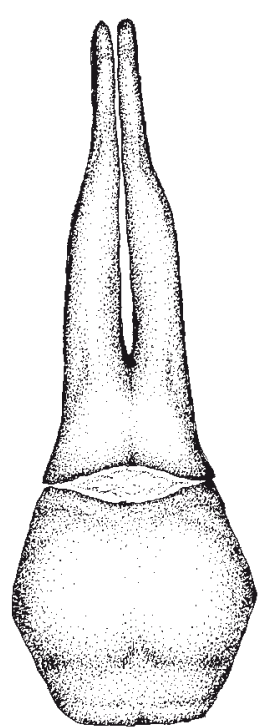

b

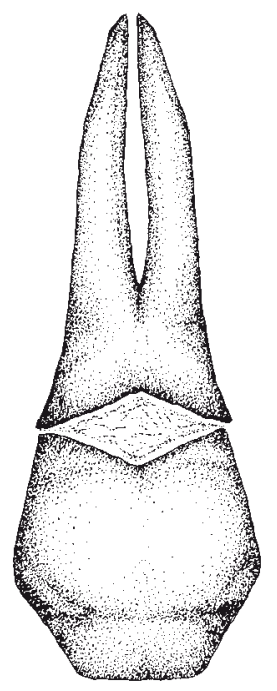

h

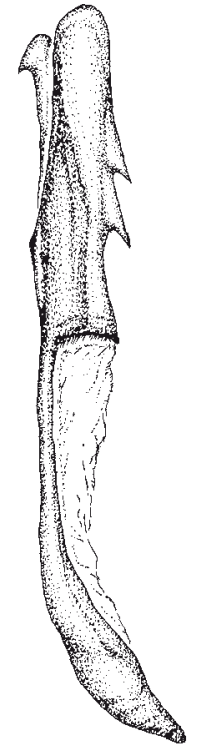

C

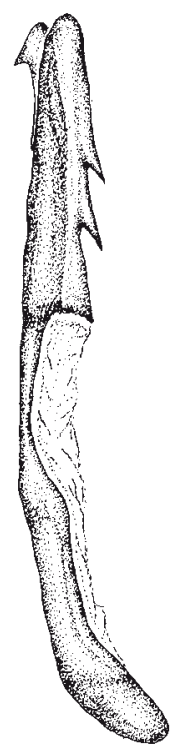

i

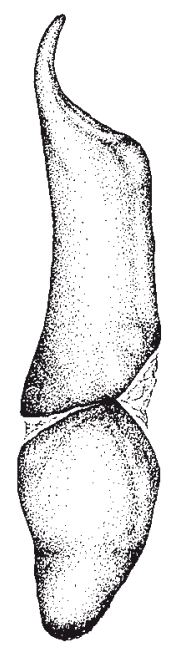

d

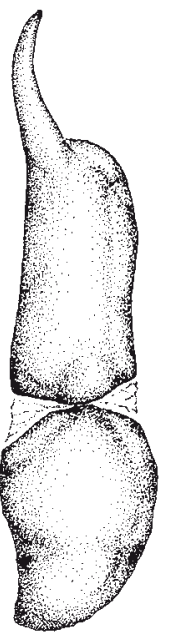

j

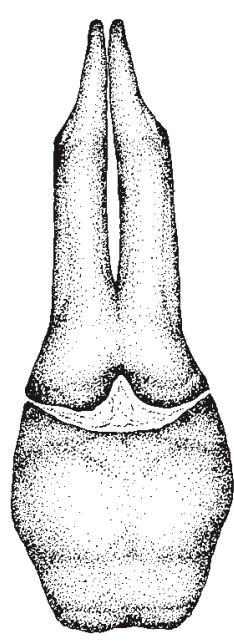

e

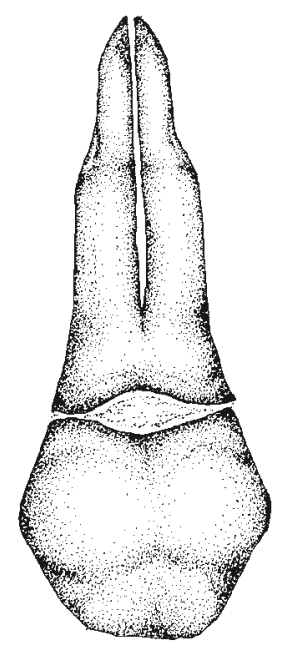

k

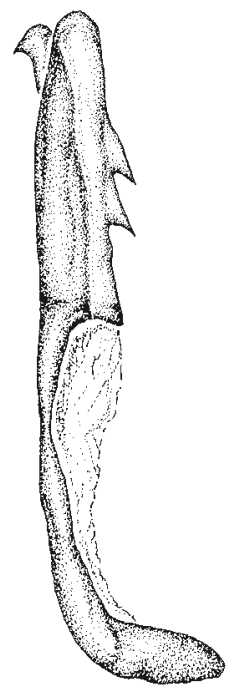

f

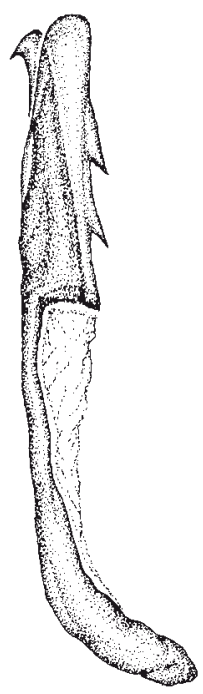

I

Fig. 6.- Genitalia masculina de Mylabris (Micrabris) deferreri (holotipo, La Sagra, Granada): a: tegmen, en visión lateral; b: tegmen, en visión dorsal; c: lóbulo medio, en visión lateral. Mylabris (Micrabris) dejeani (Venta Rasquilla, Gredos, Ávila): d: tegmen, en visión lateral; e: tegmen, en visión dorsal; f: lóbulo medio, en visión lateral. Mylabris (Micrabris) platai (Fuente de Don Manuel, Sierra Nevada, Granada): g: tegmen, en visión lateral; h: tegmen, en visión dorsal; i: lóbulo medio, en visión lateral. Mylabris (Micrabris) varians (Los Ángeles de San Rafael, Segovia): j: tegmen, en visión lateral; k: tegmen, en visión dorsal; 1 : lóbulo medio, en visión lateral. Barra de escala $=0,5$ mm. (J.L. Ruiz del.)

Fig. 6.- Male genitalia of Mylabris (Micrabris) deferreri (holotype, La Sagra, Granada): a: tegmen, lateral view b: tegmen, dorsal view; c: median lobe, lateral view. Mylabris (Micrabris) dejeani (Venta Rasquilla, Gredos, Ávila): d: tegmen, lateral view e: tegmen, dorsal view; f: median lobe, lateral view. Mylabris (Micrabris) platai (Fuente de Don Manuel, Sierra Nevada, Granada): g: tegmen, lateral view; h: tegmen, dorsal view; i: median lobe, lateral view. Mylabris (Micrabris) varians (Los Ángeles de San Rafael, Segovia): j: tegmen, lateral view k: tegmen, dorsal view; l: median lobe, lateral view. Scale bar = 0,5 mm. (J.L. Ruiz del.) 
extendidas hacia atrás alcanzan, a lo sumo, la mitad del pronoto, con los artejos IV a XI netamente más anchos y troncocónicos; por poseer en la cara externa de las tibias anteriores pelos alargados entremezclados con los tendidos; por la presencia de un proceso digitiforme en el ángulo apical externo de las protibias, ausente en el macho; y con el último esternito abdominal visible no escotado en su margen posterior.

Se ha detectado variabilidad entre los ejemplares de la serie típica en la longitud total, que oscila en los machos entre 8,5 y $12,1 \mathrm{~mm}$ (media $=$ $9,82 ; \mathrm{n}=13)$ y en las hembras entre 8,1 y $13 \mathrm{~mm}$ (media $=10,84 ; n=15)$, la media del conjunto es de $10,36 \mathrm{~mm}$. Además se han observado ligeras diferencias en la longitud de las antenas; pequeñas variaciones en la amplitud e impresión de las depresiones laterales situadas junto a las inserciones antenales y depresión media de la frente, que en algunos casos puede llegar a desaparecer; punteado de la cabeza y pronoto más o menos denso e impreso, mostrándose en general más denso y marcado cuanto mayor es el tamaño del ejemplar. El diseño cromático elitral muestra una variación relativamente acusada (Fig. 7), como es habitual en la mayor parte de las especies de Mylabris y de la tribu Mylabrini en general (e.g. Pardo Alcaide, 1950, 1954b; Bologna, 1991a; Trotta-Moreu y García-París, 2001). El 84,2 \% de los ejemplares $(\mathrm{n}=38)$ presentan la última banda negra (distal) completa, no dividida (Fig. 7 a-g); en el porcentaje restante, ésta se descompone en dos manchas redondeadas (aunque existe asimetría en algunos especímenes que presentan las manchas débilmente fusionadas en un élitro y separadas en el otro) (Fig. 7 h). La armadura genital masculina se muestra muy homogénea en los ejemplares estudiados (Fig. 6).

Los ejemplares estudiados de Galera y Puebla de Don Fadrique, no incluidos en la serie típica, muestran diferencias en el diseño cromático elitral, aunque todos se encuadran en los patrones ya señalados para la población de La Sagra (Fig. 7).

DistRIBUCIÓN Y NOTAS AUTOECOLÓGICAS: Los ejemplares colectados por J. de Ferrer se localizaron en las cercanías del "Cortijo de San José" en la carretera Huéscar-Santiago de la Espada, a una altitud de 1300-1350 m en el macizo de La Sagra (UTM: 30SWG39) (Término municipal de Huéscar, Granada) (J. de Ferrer, com. pers., 2004). El resto de los ejemplares examinados se capturaron en la Sierra de la Sagra (WH30) y en el complejo de sierras calizas que se localizan al sur de
La Sagra, en los municipios de Huéscar (WG38), Galera (WG37) y Puebla de Don Fadrique (WH40), de donde la especie ha de considerarse endémica hasta el momento.

La comunidad de Mylabrini de la Sierra de La Sagra y serranías próximas es especialmente singular, ya que además de albergar a $M$. deferreri, constituye el refugio ibérico más meridional para varias especies de amplia distribución en áreas septentrionales como Hycleus polymorphus (Pallas, 1771) (Trotta-Moreu \& García-París, 2001), Mylabris variabilis (Pallas, 1781) (col. MNCN; Ruiz \& Ávila, 1993), o M. dejeani, que junto a $M$. deferreri fueron capturados con relativa frecuencia a principios del siglo XX.

Etimología: Tenemos el honor de dedicar esta especie al excelente entomólogo especialista en Histeridae, y químico de profesión, D. Juan de Ferrer Andreu (Barcelona, 1924), colector de buena parte de los ejemplares que integran la serie típica de esta nueva especie, que tanto y tan cordial y amablemente nos ha ayudado e instruido en el desarrollo de nuestros estudios entomológicos desde hace ya muchos años. El epíteto es un genitivo y por tanto invariable.

\section{Discusión}

Mylabris deferreri se encuadra en el subgénero Micrabris, bien caracterizado por los rasgos señalados en la diagnosis (Pardo Alcaide, 1948, 1950, 1954a, 1954b, 1969; Kuzin, 1954; Bologna, 1986, 1991a).

Los representantes del subgénero Micrabris pueden integrarse en dos grandes grupos de especies, atendiendo a la presencia o ausencia de pliegue longitudinal en el lado externo de la región inferior de las protibias del macho (Pardo Alcaide, 1950, 1954b, 1975; Bologna, 1991a). Pardo Alcaide acuñó el término "androplicata" (sensu Pardo Alcaide, 1948; nombre sin validez nomenclatural, no disponible; véase Bologna, 1991a; Bologna \& Pinto, 2002) para denominar al conjunto de taxones específicos que ostentan el referido carácter. Las especies ibéricas del subgénero Micrabris con pliegue longitudinal en el lado externo de la región inferior de las protibias del macho son Mylabris (Micrabris) dejeani Gyllenhal, 1817, M. (M.) sobrina Graells, 1849, M. (M.) nevadensis (Escalera, 1915), M. (M.) maculosopunctata Graells, 1858 y M. (M.) pauper (Escherich, 1899); quedando integradas en el grupo que no presentan 

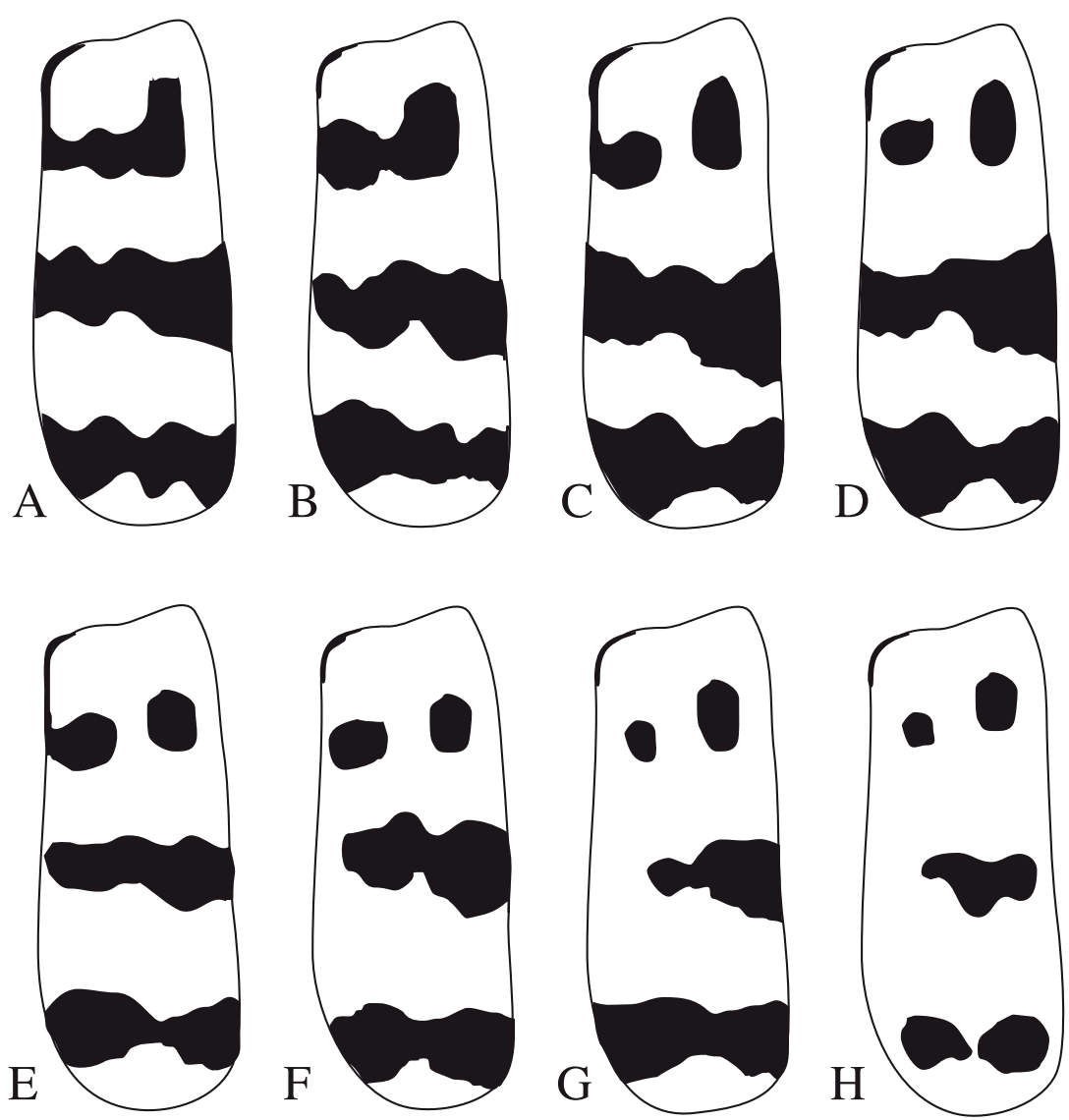

Fig. 7.- Variabilidad en el diseño del élitro derecho de Mylabris (Micrabris) deferreri. El diseño más común corresponde al patrón $\mathrm{F}$ con pequeñas variantes en la anchura y extensión de las manchas. La frecuencia $(\mathrm{n}=38)$ de cada diseño es: A: 5,3 \%; B: 2,6 \%; C: 10,5 \%; D: 10,5 \%; E: 5,3 \%; F: 44,3\%; G: 5,3\%; H: 15,8\%. Se encontraron ejemplares con diseños C, D, F y H tanto en La Sagra como en Galera. Los ejemplares de Puebla de Don Fadrique únicamente presentaron el diseño F, mientras que los diseños A, B y G, se encontraron únicamente en los ejemplares de La Sagra.

Fig. 7.- Variability on the right elytral pattern in Mylabris (Micrabris) deferreri. The commonest pattern correspond to F, with minor variants in spot width and size. Frequency $(n=38)$ of each pattern: A: $5.3 \%$; B: $2.6 \%$; $: 10.5 \%$; D: $10.5 \%$; E: $5.3 \%$; F: $44.3 \%$; G: $5.3 \%$; H: $15.8 \%$. We found specimens with pattern C, D, F and H both at La Sagra and Galera. Specimens from Puebla de Don Fadrique presented pattern F, while pattern A, B and G, were found only in specimens from La Sagra.

el citado pliegue las siguientes: Mylabris (Micrabris) varians Gyllenhal, 1817, M. (M.) flexuosa Olivier, 1811, M. (M.) platai Pardo Alcaide, 1975 (Pardo Alcaide, 1948, 1950, 1975) y la especie descrita en este trabajo, M. (M.) deferreri.

Sin embargo, el aludido caracter dimórfico sexual es, en ocasiones, de difícil apreciación (sobre todo en ejemplares de pequeño tamaño; obs. pers.), a la vez que inválido para la adscripción de hembras a uno u otro grupo de especies. Alternativamente, las especies ibéricas de Micrabris pueden separarse fenéticamente por la conformación del punteado y pilosidad de la base de los élitros. Así, un primer conjunto, que integra a $M$. sobrina, $M$. nevadensis, $M$. maculosopunctata, M. pauper y $M$. flexuosa, se caracterizaría por presentar la base elitral con un punteado similar o muy poco distinto de el del resto del élitro, siempre fuerte y espaciado, en el que se inserta una pubescencia alargada y bien visible (Pardo Alcaide, 1950, 1975; Bologna, 1991a). El segundo conjunto, que reúne a $M$. dejeani, $M$. varians, $M$. platai y M. deferreri presenta la base de los élitros con un punteado y pilosidad muy distinta de 
la del resto de la superficie elitral. El punteado de la base de los élitros es muy fino y apretado, en ocasiones prácticamente inexistente, y la pubescencia muy corta, densa y erecta, a veces casi imperceptible incluso examinando el ejemplar en visión lateral, y en algunos casos con la pubescencia caduca (Pardo Alcaide, 1950, 1975; Bologna, 1991a). Según Bologna (1991b) es probable que este tipo singular de punteado y pilosidad (microsetas erectas), se encuentre relacionado con un modelo de cortejo sexual frontal, observado en $M$. (Micrabris) pusilla Olivier, 1811, único en el seno de la tribu Mylabrini, donde las microsetas y los poros cuticulares (punteado) podrían estar implicados en la recepción de estímulos táctiles y emisión de feromonas de contacto.

Mylabris deferreri se separa de $M$. dejeani porque ésta presenta los élitros con un punteado muy fino en la base hasta el nivel de la banda negra anterior, desde donde el punteado se va haciendo paulatinamente más grueso hasta la región apical, con la pubescencia basal densa, muy corta y erecta, mientras que en $M$. deferreri el punteado basal es aún más fino y la pubescencia que en él se inserta más densa, cortísima y erizada; el punteado de cabeza y pronoto es menos fuerte y denso en M. dejeani, con la separación entre los puntos similar al diámetro de los mismos, mientras que en M. deferreri dicho punteado es más fuerte y denso, con los puntos subcontiguos; los élitros de $M$. dejeani presentan la mancha negra externa de la primera serie (anterior) alargada longitudinalmente, y generalmente alcanza el callo humeral, y la interna remonta por la sutura elitral hasta llegar al escutelo, mientras que en $M$. deferreri la externa, que en ocasiones se une a la interna formando una banda sinuosa, no alcanza la base del húmero y la interna sólo en contadas ocasiones alcanza el escutelo a través de la sutura, pero en estos casos la línea negra que bordea dicha sutura es más estrecha que en $M$. dejeani (véase Valladares Díez, 1984: 107, fig. 13; Bologna, 1991a: 270, fig. 97); los machos de $M$. dejeani poseen un pliegue longitudinal en las protibias, ausente en M. deferreri; y, por último, los respectivos edeagos son comparativamente distintos (Fig. 6). Tras el examen de tres ejemplares de $M$. dejeani procedentes de Galera (Granada) (Escalera leg., 1900. Col. MNCN) podemos señalar que ambas especies se encuentran localmente en simpatría, aunque a rasgos generales $M$. dejeani presenta una distribución más septentrional (e.g. Górriz, 1882; Oliveira, 1894; Champion, 1903, 1904; López-Neyra, 1914; Fuente, 1933; Pardo Alcaide, 1950; Valladares y
Salgado, 1983; Valladares Díez, 1984; Bologna, 1991a; Recalde et al., 2002; Pérez-Moreno et al., 2003).

Mylabris platai, taxon que al igual que $M$. deferreri carece de pliegue en las protibias del macho, se separa de éste principalmente por presentar el punteado del pronoto finísimo y contiguo, con la pilosidad que en él se inserta también muy densa y corta; el diseño cromático elitral de M. platai, presenta la serie posterior constituida por dos puntos que a menudo se unen parcialmente (véase Pardo Alcaide, 1975: 48, fig. 3), mientras que en $M$. deferreri la serie posterior casi siempre está constituida por una banda ancha, excepto en contadas ocasiones en los que se diferencian las dos manchas terminales (Fig. 7). Los edeagos de ambos taxones se diferencian con claridad (Fig. 6).

Mylabris deferreri es similar a $M$. varians, tanto en lo concerniente a su morfología externa como al patrón cromático elitral. La morfología y punteado de cabeza y pronoto son semejantes en ambos táxones. El punteado de la base de los élitros, aunque muy fino, es más patente en $M$. deferreri, al igual que la pilosidad que en él se inserta, si bien la intensidad con la que se muestra este carácter está sujeta a cierta variabilidad, por lo que no puede ser adoptado como un rasgo distintivo válido. En cuanto al diseño elitral, $M$. varians es extremadamente variable, habiéndose descrito numerosas variedades o aberraciones (véase e.g. Chevrolat, 1865; Marseul, 1870; Fuente, 1909; Pic, 1909; Soumakov, 1915; Kaszab, 1958; Valladares Díez, 1984), con un patrón general constituido por tres series de manchas negras (2, $2,2)$ más o menos amplias que pueden unirse formando bandas, fundamentalmente los de la serie media, o bien desaparecer, especialmente los de la serie anterior y el punto interno de la serie mediana (vease Valladares Díez, 1984: 109, fig, 15). Sin embargo, en esta especie, como norma general los puntos de la serie posterior se encuentran separados y no forman banda, de hecho, de entre las diez variedades que señala Fuente (1909), ninguna presenta una banda posterior, al igual que se observa en la iconografía que al respecto ofrece Valladares Díez (1984); no obstante, entre el abundante material de comparación estudiado (Ruiz y GarcíaParís, en prep.) se han encontrado algunos ejemplares con dicha banda presente, estrechada en el medio y constituida por la unión de los dos puntos de la serie posterior. En M. deferreri la banda posterior es constante en la casi totalidad de los ejemplares estudiados y generalmente más 
ancha que en los pocos individuos de $M$. varians que la poseen; la banda media es igualmente constante, aunque en ciertos especímenes ésta no llega a alcanzar los márgenes laterales del élitro. En general, el patrón cromático de $M$. deferreri es más bandeado que en $M$. varians, con individuos en los que las tres series negras conforman bandas enteras, caso que no se conoce en $M$. varians. Para separar sin dudas estas dos especies es necesario acudir al examen de la armadura genital masculina (Fig. 6). Mylabris deferreri presenta un edeago con la vaina parameral muy alargada y con los lóbulos distales igualmente alargados, muy poco curvados y de aspecto digitiforme en visión dorsal; y los ganchos del lóbulo medio están más próximos entre sí que en $M$. varians.

Si bien Fuente (1933) y Pardo Alcaide (1950) atribuyen a $M$. varians una distribución general que concierne a toda la Península Ibérica, el grueso de citas se centran en el sector centro-occidental de la misma (Martínez Sáez, 1873; Górriz, 1881, 1882, 1902; Oliveira, 1894; Fuente, 1909, 1933; López-Neyra, 1914; Redondo, 1915; Torres Sala, 1962; Valladares y Salgado, 1983; Valladares Díez, 1984; Recalde et al., 2002; Pérez-Moreno et al., 2003), aunque parte de estos registros han de ser considerados con cautela debido a la confusión reinante entre los Micrabris ibéricos. Entre éstos últimos han de considerarse los únicos dos registros andaluces de $M$. varians, referentes ambos a "Granada" (Rosenhauer, 1856; Fuente, 1933), que casi con seguridad, como ya comenta Pardo Alcaide (1975), corresponden a $M$. platai. Mylabris varians y $M$. deferreri parecen mostrar áreas de distribución alopátricas.

Por proximidad geográfica en la región béticorifeña, la única especie de Micrabris del Magreb, Mylabris (Micrabris) djebelina Pic, 1902, distribuida por las regiones montañosas de Marruecos y oeste de Argelia (e.g. Pardo Alcaide, 1954a, 1954b; Kocher, 1956), se separa sin dudas de $M$. deferreri, entre otros caracteres, por presentar el punteado de la base de los élitros fuerte y apenas distinto de el del resto de la superficie elitral y la pubescencia de esta región larga y bien visible (Pardo Alcaide, 1954a, 1954b; obs. pers.).

Desde un punto de vista corológico, $M$. deferreri integra, junto con $M$. nevadensis y $M$. platai, un pequeño grupo de Micrabris restringido al macizo bético. Estas especies son los endemismos ibéricos de Mylabris con áreas de distribución más reducidas; la primera, hasta el momento, exclusiva de La Sagra y zonas adyacentes (Granada), la segunda de las partes altas de Sierra
Nevada (Escalera, 1915; Pardo Alcaide, 1950), y la tercera de Sierra Nevada, Sierra de Gádor y Sierra de Filabres (Pardo Alcaide, 1975; Ruiz y Ávila, 1993).

\section{AGRADECIMIENTOS}

Agradecemos a Juan de Ferrer su amabilidad al cedernos sus Mylabrini para estudio, a Nuria Trotta Moreu su ayuda en la preparación de genitalias, a Javier Pérez-López el remitirnos, hace ya algunos años, uno de los ejemplares que integran la serie típica, a Marco Bologna y Miguel Ángel Alonso Zarazaga la revisión crítica del manuscrito, a Mercedes París y Carolina Martín las facilidades prestadas para la revisión de la colección del MNCN y al Instituto de Estudios Ceutíes por el apoyo que viene prestando a uno de los autores (JLR). Este trabajo se ha beneficiado de los recursos del proyecto de la DGI: CGL2004-04680-C10-10/BOS.

\section{Referencias}

Bologna, M. A., 1979. Meloidae di Turchia. I. Contributo (Coleoptera). Fragmenta Entomologica, 15: 143-199.

BolognA, M. A., 1986. Nota tassonomica su alcune Mylabris del gruppo geminata (Coleoptera, Meloidae). Bollettino dell Museo Regionale di Scienze naturali di Torino, 4 (1): 291-310.

Bologna, M. A., 1991a. Fauna d'Italia. XXVIII. Coleoptera Meloidae. Calderini. Bologna. 541 pp.

Bologna, M. A., 1991b. Note sul comportamento sessuale nella tribu Mylabrini e descrizione di un nuovo modello di corteggiamento (Coleoptera, Meloidae). Bollettino dell'Associazione Romana di Entomologia, 45 [1990]: 51-56.

Bologna, M. A., 1994. I Meloidae della Grecia (Coleoptera). Fragmenta Entomologica, 25. Supplemento: 1-119.

Bologna, M. A., Cobolli Sbordoni, M., De Mattheis, E. \& Mattocia, M., 1988. Divergenza genetica tra popolazioni sud europee di Mylabris flexuosa Olivier (Coleoptera, Meloidae). Atti XV Congresso nazionale italiano di Entomologia, L'Aquila, 1988: 673-688.

Bologna, M. A. \& Coco, E., 1991. Revisione del genere Croscherichia Pardo Alcaide, 1950 (Coleoptera, Meloidae). Memorie della Società Entomologica italiana, 69 [1990]: 97-180.

Bologna, M. A. \& Pinto, J. D., 2002. The Old World genera of Meloidae (Coleoptera): a key and synopsis. Journal of Natural History, 36: 2013-2102.

Champion, G. C., 1903. An entomological excursion to Bejar, Central Spain. Transactions of the Entomological Society of London, 1903: 165-182.

Champion, G. C. 1904. An entomological excursion to Moncayo, N. Spain. Transactions of the Entomological Society of London, 1904: 81-99. 
Chevrolat, L. A., 1865. Descriptions de coléoptères d'Espagne, nouveaux ou peu connus. $1^{\mathrm{a}}$ mémoire. Revue et Magasin de Zoologie, (2) 17: 390-397.

Escalera, M. M. DE LA, 1915. Una nueva especie de Zonabris de España. Boletín de la Real Sociedad española de Historia natural, 15: 493-494.

Fuente, J. M. DE LA, 1909. Datos para la fauna de la provincia de Ciudad Real. Boletín de la Real Sociedad española de Historia natural, 9: 306-310.

Fuente, J. M. DE LA, 1933. Meloidae. Catálogo sistemático geográfico de los Coleópteros observados en la Península Ibérica, Pirineos propiamente dichos y Baleares. Boletín de la Sociedad entomológica de España, 16: 18-32; 45-49.

GóRRIZ, R. J., 1881. Nuevas observaciones sobre costumbres y metamorfosis de algunos vesicantes. Actas de la Sociedad Española de Historia natural, 10: $59-64$.

GóRRIZ, R. J., 1882. Ensayo para la Monografía de los coleópteros Meloideos indígenas con aplicación a las ciencias médicas. Sanz y Navarro. Zaragoza. 201 pp.

GóRriz, R. J., 1902. Coleópteros de la Cuenca del Ebro. Boletín de la Sociedad aragonesa de Ciencias naturales, 1: 180-186.

HEYDEN, L. vON, 1870. Entomologische reise nach dem südlichen Spanien, der Sierra de Guadarrama und Sierra Morena, Portugal und den Cantabrischen Gebirgen, mit Bescreibungen der neuen Arten. Herausgegeben von dem entomologischen Vereine. Nicolai'sche Verlagsbuchhandlung. Berlin, Primera parte: 1-175.

KASZAB, Z., 1958. Neue Meloiden-namen (Coleoptera). Annales historico-naturales Musei nationalis hungarici (s.n.), 50: 189-191.

KASZAB, Z., 1969. The system of the Meloidae (Coleoptera). Memorie della Società Entomologica Italiana, 48: 241-248.

Kocher, L., 1956. Catalogue commenté des Coléoptères du Maroc. V. Héteromères (Tenebrionides excepts). Travaux de l'Institute Scientifique Chérifien (série zoologie), 10: 1-107.

KuZIN, V. S., 1954. K posnanyiu systemy narybnikov (Coleoptera, Meloidae, Mylabrini). Trudy Vsesoyuznogo Entomologischeskogo Obshchetsva, 44: 336-379.

LóPEZ-NEYrA, C. R., 1914. Claves dicotómicas para la determinación de los meloidos indígenas. Boletín de la Real Sociedad Española de Historia Natural, 14: 461-475.

Marseul, S. A. DE, 1870. Monographie des Mylabrides d'Europe et des contrées limitrophes en Afrique et en Asie. L'Abeille, 7(2 $2^{\text {ème }}$ partie): 1-204.

Martínez SÁEz, F. DE P., 1873. Datos sobre algunos coleópteros de los alrededores de Cuenca. Anales de la Sociedad Española de Historia natural, 2: 53-75.
OliveIRA, M. P. DE, 1894. Catalogue des Insectes du Portugal. Coléoptères. Imprensa da Universidade. Coimbra. 393 pp.

Pardo Alcaide, A., 1948. Estudios sobre Meloidae. I. Acerca de la validez específica de Mylabris maculoso-punctata Grll., rosinae Escher. y pauper Escher. Eos, 24: 493-502.

Pardo Alcaide, A., 1950. Estudios sobre Meloidae. II. Los "Mylabrini" de la Península Ibérica. Boletín de Patología Vegetal y Entomología agrícola, 17 [1949]: 61-82.

Pardo Alcaide, A., 1954a. Estudios sobre Meloidae. IV. Sobre algunos Mylabris Fab. Del occidente norteafricano. (Primera parte). Eos, 30: 331-344.

Pardo Alcaide, A., 1954b. Études sur les Meloidae. V. Les Mylabrini du Maroc et du Sahara occidental espagnol (Col. Meloidae). Bulletin de la Société des Sciences Naturelles et physiques du Maroc, 34: 55-88.

Pardo Alcaide, A., 1969. Études sur les Meloidae. XXI. Matériaux pour une révision des Mylabrini de l'Afrique du nord et du Moyen Orient ( $2^{\mathrm{a}}$ partie). Eos, 44: 367-376.

Pardo Alcaide, A., 1975. Estudios sobre Meloidae (Coleoptera). XXIV. Una nueva especie de Mylabris Fabricius (Mylabris platai n. sp.) de Sierra Nevada. Cuadernos de Ciencias Biológicas, 4 (1): 45-49.

Pérez-Moreno, I., San Martín, A. F. \& Recalde, J. I., 2003. Aportaciones corológicas y faunísticas sobre meloidos ibéricos (Coleoptera: Meloidae). Boletín de la Sociedad Entomológica Aragonesa, 33: 195-217.

PIC, M., 1909. Descriptions ou diagnoses et notes diverses. (Suite). L'Échange, Revue Linnéenne, 298: 169171.

Recalde, J. I, San Martín, A. F. \& Pérez-Moreno, I., 2002. Meloidae. Catalogus entomofauna aragonesa, 26: 3-21.

Redondo, A., 1915. Coleópteros de Salamanca. Brotéria, Série Zoología, 1915: 14-48.

Rosenhauer, W. G., 1856. Die Thiere Andalusiens nach dem Resultate einer Reise zusammengesetellt, nebst den Beschreibungen von 249 neuen oder bis jetzt noch unbeschriebenen Gattungen und Arten. Verlag von Theodor Blaesing. Erlangen. 429 pp.

Ruiz, J. L. \& Ávila, J. M., 1993. Contribución al conocimiento de los Meloidae (Coleoptera) en el sur de la Península Ibérica. Boletín de la Asociación española de Entomología, 17: 141-148.

Soumakov, G., 1915. Les espèces paléarctiques du genre Mylabris Fabr. (Coleoptera, Meloïdae). Horae Societatis Entomologicae Rossicae, 42(1): $1-73$. 
Torres-SAla, J., 1962. Catálogo de la colección entomológica "Torres-Sala" de coleópteros y lepidópteros de todo el mundo. Institución Alfonso el Magnánimo. Diputación Provincial de Valencia. Valencia. Vol. I. 487 pp.

Trotta-Moreu, N. \& García-París, M., 2001. Los Hycleus Latreille, 1829 (Coleoptera, Meloidae) de la Península Ibérica: distribución geográfica y variabilidad elitral. Graellsia, 57 (1): 99-111.

Valladares Díez, L. F., 1984. Estudio de los coleópteros vesicantes de la provincia de León (Coleoptera, Meloidae). Institución "Fray Bernardino de Sahagún". Diputación Provincial de Aragón. Consejo Superior de Investigaciones Científicas. León. 156 pp.
Valladares, L. F. \& Salgado, J. M., 1983. Contribución al conocimiento de los Meloidae (Col.) En la provincia de León. Boletín de la Asociación española de Entomología, 7: 79-100.
Recibido, 9-VIII-2004

Aceptado, 23-XI-2004 Publicado, 31-XII-2004 